Research Article

\title{
Chaos suppression in fractional order systems using state-dependent noise
}

\author{
A. O. Adelakun ${ }^{1}$. S. T. Ogunjo' ${ }^{1} \cdot$ I. A. Fuwape ${ }^{1,2}$
}

Received: 18 June 2019 / Accepted: 31 October 2019 / Published online: 13 November 2019

(c) Springer Nature Switzerland AG 2019

\begin{abstract}
Noise play a creative role in the evolution of periodic and complex systems which are essential for continuous performance of the system. The interaction of noise generated within one component of a chaotic system with other component in a linear or nonlinear interaction is crucial for system performance and stability. These types of noise are inherent, natural and insidious. This study investigates the effect of state-dependent noise on the bifurcation of two chaotic systems. Circuit realization of the systems were implemented. Numerical simulations were carried out to investigate the influence of state dependent noise on the bifurcation structure of the Chen and Arneodo-Coullet fractional order chaotic systems. Results obtained showed that state dependent noise inhibit the period doubling cascade bifurcation structure of the two systems. These results pose serious challenges to system reliability of chaotic systems in control design, secure communication and power systems.
\end{abstract}

Keywords Noise · Fractional order · Chaos bifurcation · Chen system

PACS $05.45 . \mathrm{Pq} \cdot 05.45 . \mathrm{Gg} \cdot 05.45 . \mathrm{Ac}$

\section{Introduction}

Fractional order models are best suited to capture the memory effects in mathematical models [1]. The lack of appropriate methods of solution has made fractional order systems unattractive to scientists. However, in recent times, adequate mathematical representation and solutions has created a resurgence and renewed interest in the subject of fractional order. The order of fractional derivative is an index of memory [2] and as stable as their integer order counterpart [3]. Due to their relevance, fractional order models have been considered in finance [4], psychology [5] and electronic systems [6].

Noise is ubiquitous and inherent in nature. The role of noise in a system depends on how it was introduced and intended function of the system. Noise is desirable in some systems while it is unwanted in others. The threshold of chaos can be increased or reduced through noise perturbations [7] and synchronization of chaotic oscillators with noise [8]. Chaos suppression due to noise has been reported in the Duffing oscillator [9] , parametrically driven Lorenz system [10], Belousov-Zhabotinsky reaction [11] and logistic map [12]. Dennis et al. [13] suggests that noise cannot induce chaos. However, the introduction of noise has also been found to enhance synchronization of chaotic systems [14,15] and induce chaos in systems [16-18]. Gao [19] showed that noise can induced chaos when the noise level is within a narrow range. It has been posited that real life neurons are robust to noise [20].

The possibility of generating noise from chaotic systems has been considered. The perceived similarity between chaos and random numbers make chaos a suitable

S. T. Ogunjo, stogunjo@futa.edu.ng| 'Department of Physics, Federal University of Technology Akure, Akure, Ondo State, Nigeria. ${ }^{2}$ Michael and Cecilia Ibru University, Ughelli North, Delta State, Nigeria. 
Table 1 Statistical properties of state-dependent noise considered

\begin{tabular}{|c|c|c|c|c|c|c|}
\hline & \multicolumn{3}{|c|}{ Chen system } & \multicolumn{3}{|c|}{ Arneodo-Coullet system } \\
\hline & $U_{1}$ & $U_{2}$ & $\xi\left(U_{1}, U_{2}\right)$ & $U_{1}$ & $U_{2}$ & $\xi\left(U_{1}, U_{2}\right)$ \\
\hline Mean & 0.8683 & 0.8808 & -0.0042 & 1.8461 & 0.0809 & 0.0051 \\
\hline Median & 1.6761 & 1.8132 & 0.3603 & 1.9403 & 0.3346 & 0.3774 \\
\hline STD & 7.6955 & 8.3352 & 1.6158 & 0.8544 & 1.7650 & 0.9030 \\
\hline Skewness & -0.1833 & -0.1776 & 0.0524 & -0.3723 & -0.4250 & 0.1719 \\
\hline
\end{tabular}

candidate to realize truly random numbers. Murch and Bates [21] proposed a mechanism for generating coloured noise through by hierarchies of nonlinear recursive loops. This was extended to study the performance of random noise generated by bounded and unbounded chaos with a gaussian noise [22]. A chaotic digital phase-locked loop system has also been developed to generate pseudo-random noise [23]. The successful generation of large scale chaos random number generator was implemented using Duffing oscillators [24]. Random numbers generated from chaotic sequences have been employed in video encryption [25]. By using shifters and multiplexers, the phase space from the Lorenz and Lu systems have been utilized to generate random numbers on Field programmable gate arrays [26].

The role of different types of noise has been investigated on integer and discrete chaotic systems. There is the need to understand the dynamic influence of noise on the memory effect of fractional order chaotic systems. Since memory is an intrinsic property of a system, it is intuitive to understudy the interaction of noise generated within the system states and the system itself. Previous researches have considered the role of external noise on the dynamics of chaotic system. Although external noises play a significant role, the impact
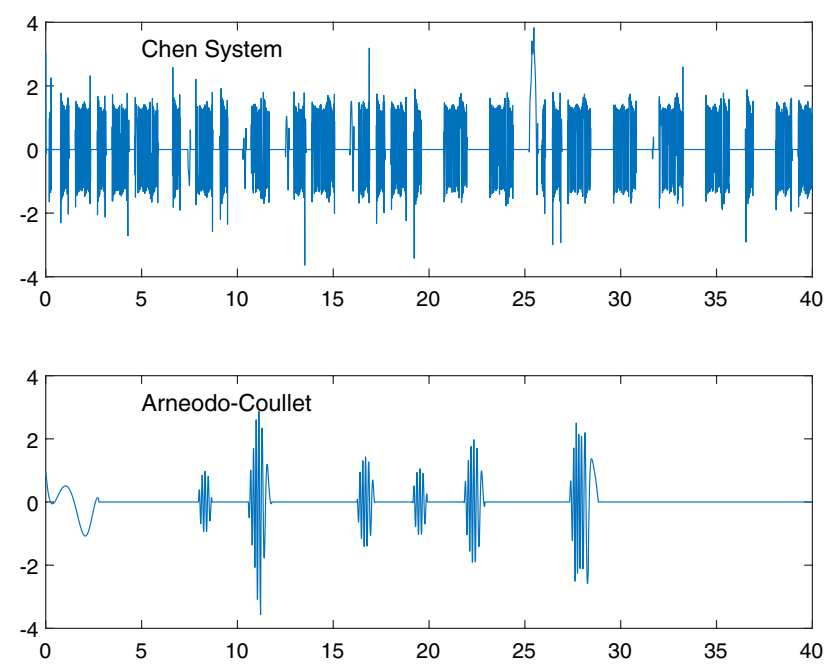

Fig. 1 State dependent noise for (top figure) the Chen system with system parameters $(a, b, c)=(40,3,28)$ using fractional order 0.9 (bottom figure) the Arneodo-Coullet system with parameters $(e, f)=(65.0)$ using fractional order 0.9 of intrinsic noise cannot be ruled out. In our approach, we aim to generate noise from within a chaotic system and feed the same noise back into the system. This approach is different from the other approaches which uses external noise or generate noise from a chaotic system but do not consider its effect as feedback noise. This research is motivated by the need to understand noise generation by fractional order systems and the influence of the generated noise as a feedback into the system. The aim of this study is to investigate the impact of state-dependent noise in a class of fractional order chaotic systems. The potential of chaotic system in secure communication makes this study important. It will provide information to systems designer on the role of noise in the system, as well as, the impact of internally generated noise on system performance. In Sect. 2, the concept and definition of fractional order systems, state dependent noise, as well as, systems to be considered are introduced. In Sect. 3, we discuss the result of our analysis while our conclusions are presented.

\section{Methodology}

\subsection{Fractional order derivatives}

Fractional derivatives have been defined in different ways [27]. The Caputo's definition of fractional order differ-integral equations are given as

${ }_{a}^{C} D_{t}^{\alpha} f(t)=\frac{1}{\Gamma(\alpha-m)} \int_{a}^{t} \frac{f^{(m)} \tau}{(t-\tau)^{\alpha+1-m}} d \tau$

where $m-1<\alpha \leq m \in \mathbb{N}$ and $\alpha \epsilon \mathbb{R}$ is a fractional order of the differ-integral of the function $f(t)[28,29]$.

Another common definition is Riemann-Liouville, which can be denoted as:

$\frac{d^{\alpha} f(t)}{d t^{\alpha}}=\frac{1}{\Gamma(n-\alpha)} \frac{d^{n}}{d t^{n}} \int_{0}^{t} \frac{f(t)}{(t-\tau)^{\alpha-n+1}} d \tau$

In Eq. 2, $\Gamma(\cdot)$ is the gamma function, where $n-1<\alpha<n$ and $n$ is an integer. The Laplace transform of Eq. 2 can be written as

\section{SN Applied Sciences}




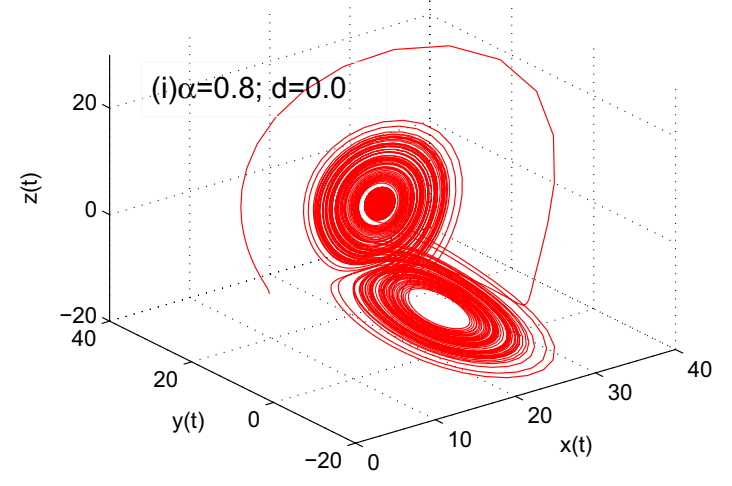

(a)

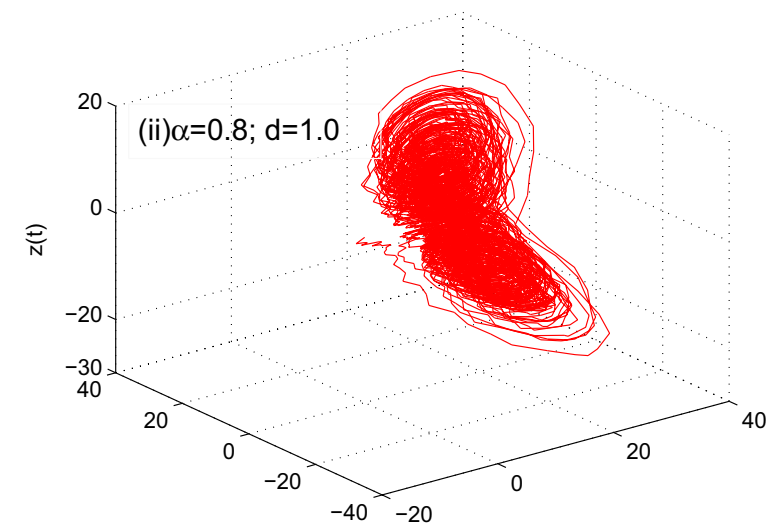

(b)

Fig. 2 3D Phase space representation of system 8 with fractional order $\alpha=0.8$ and noise level, $d$ a $0.0 ;$ and $\mathbf{b} 1.0$

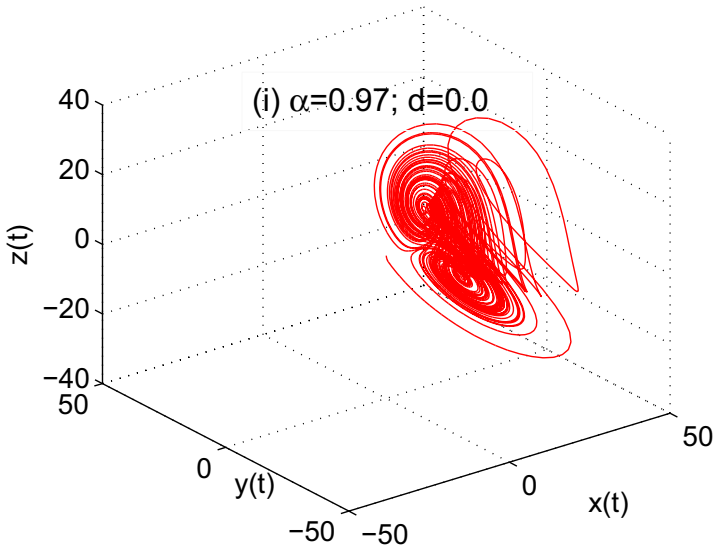

(a)

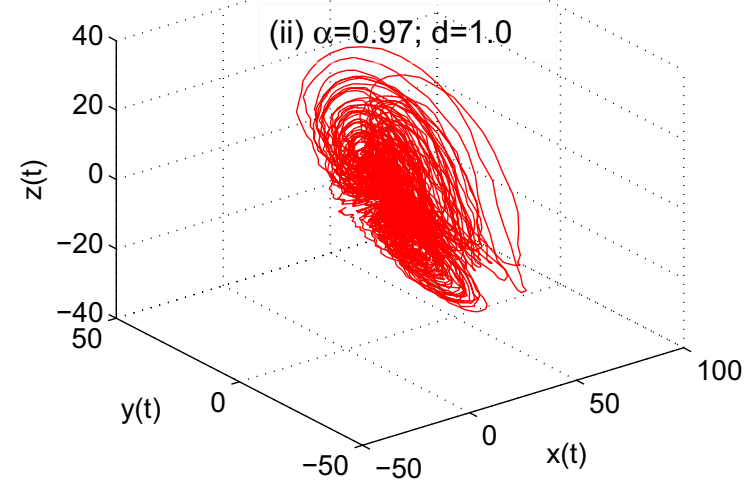

(b)

Fig. 3 3D Phase space representation of system 9 with fractional order $\alpha=0.97$ and noise level, $d$ a 0.0 ; and $\mathbf{b} 1.0$

$$
L\left\{\frac{d^{\alpha} f(t)}{d t^{\alpha}}\right\}=s^{q} L\{f(t)\}-\sum_{k=0}^{n-1} s^{k}\left\{\frac{d^{\alpha-1-k} f(t)}{d t^{\alpha-1-k}}\right\}_{t=0}
$$

If the initial conditions are zero for Eq. 3, it implies the Riemann-Liouville fractional derivative is

$L\left\{\frac{d^{\alpha} f(t)}{d t^{\alpha}}\right\}=s^{q} L\{f(t)\}$

However, the transfer function $F(s)$ for the fractional integral operator of order $\alpha$ can therefore be expressed as $F(s)=\frac{1}{s^{q}}$ in the frequency domain. With approximation approach, the errors of $2 \mathrm{~dB}$ have been deduce for fractional integral operator $\frac{1}{s^{\alpha}}$ [30]. Fractional order systems are said to be commensurate if the orders are the same (ie $\alpha_{1}=\alpha_{2}=\cdots=\alpha_{n}=\alpha$ ).

\subsection{State dependent noise}

We define a state-dependent noise as feedback noise arising from one or more components of the system. Consider a system defined as $f(x, y, z)$. If a fraction of the output $y$ is made to interfere with the input $x$, a state-dependent noise has been introduced. This state-dependent noise can be a linear or nonlinear function of the state variables. Box and Muller [31] proposed that using Eq. 5, two random number streams $\left(U_{1}\right.$ and $\left.U_{2}\right)$ can be used to generate standard random numbers. In this study, we consider 
Fig. 4 Bifurcation diagram of the Chen system (Equation 8) with fractional order $\alpha=0.97$ and noise level, $d$ (top) 0.0 ; and (bottom) 1.0
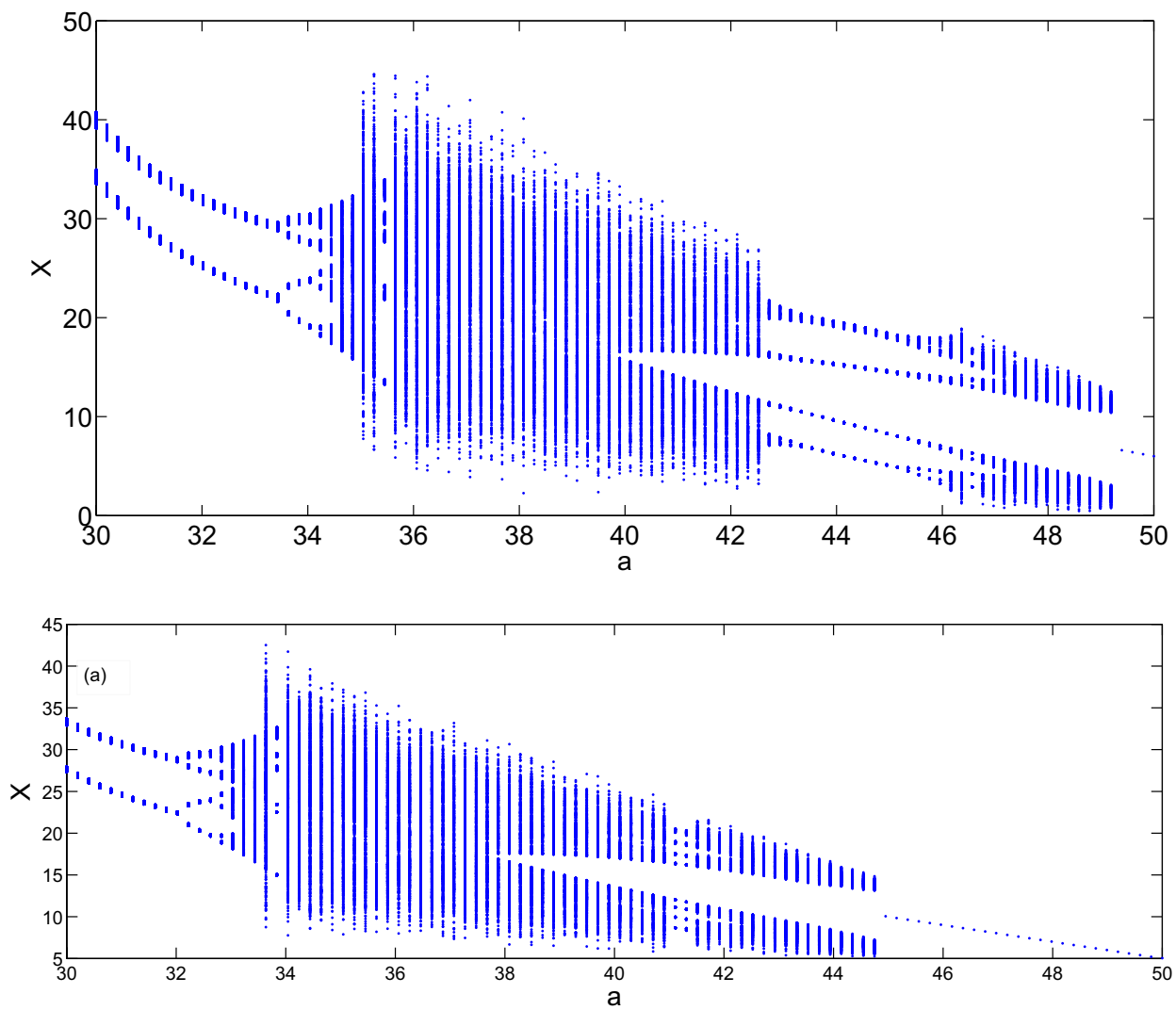

two systems $f(x, y, z)$ where the state $z$ is perturbed with feedback noise which is a nonlinear function of $x=U_{1}$ and $y=U_{2}$ states.

$\xi\left(U_{1}, U_{2}\right)=d\left(\sqrt{\left(-2 \log U_{1}\right)} \cos 2 \pi U_{2}\right)$

where $d$ is the noise strength. If the states of the system are independently chaotic, the noise $\xi(x, y)$ can be regarded as a chaotic noise. The statistical properties of $\xi\left(U_{1}, U_{2}\right)$ are shown in Table 1 and the time series in Fig. 1.

\subsection{Systems}

The Chen and Ueta [32] and Arneodo et al. [33] chaotic systems were considered in this study. The integer order Chen system is defined as $\dot{x}=a(y-x)$

$\dot{y}=(c-a) x-x z+c y$

$\dot{z}=(x y-b z)$

This system has been shown to be chaotic when $a=35$, $b=3$, and $c=28$. Chen system is related to but not topological equivalent to either the Lorenz [34] or Rössler [35] chaotic systems.

The Arneodo-Coullet chaotic system is defined as

$\dot{x}=y$

$\dot{y}=z$

$\dot{z}=e x-f y-z-x^{3}$

The fractional order form of the Chen and Arneodo-Coullet systems with continuous time, state-space noise (Sect. 2.2) are given in Eqs. 8 and 9 respectively. 

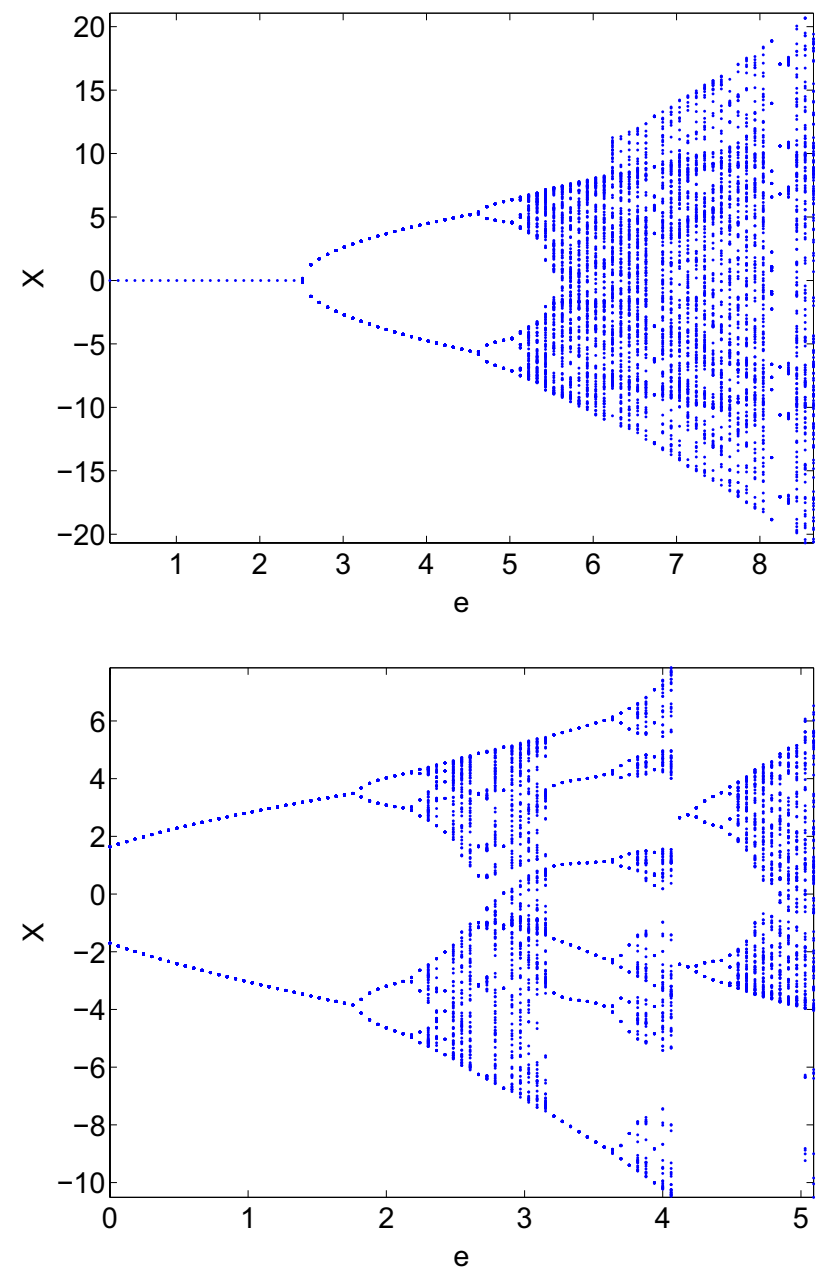

Fig. 5 Bifurcation diagram of the Arneodo-Coullet system (Equation 9) with fractional order $\alpha=0.97$ and noise level, $d$ (top) 0.0; and (bottom) 0.5

$$
\begin{aligned}
& \frac{d^{\alpha} x}{d t^{\alpha}}=a(y-x) \\
& \frac{d^{\alpha} y}{d t^{\alpha}}=(c-a) x-x z+c y \\
& \frac{d^{\alpha} y}{d t^{\alpha}}=(x y-b z)+\xi(x, y) \\
& \frac{d^{\alpha} x}{d t^{\alpha}}=y \\
& \frac{d^{\alpha} y}{d t^{\alpha}}=z \\
& \frac{d^{\alpha} z}{d t^{\alpha}}=e x-f y-z-x^{3}+\xi(x, y)
\end{aligned}
$$

The constants are given as $a=35, b=3, c=28, e=5.5$ and $f=5.0$ for the two systems. The circuit realization and phase space for the two fractional order systems are shown in "Appendix A".

\section{Results and discussion}

Numerical simulations of systems (8) and (9) using different fractional order, $\alpha$ and noise level were implemented. Using fractional orders of 0.8 (Fig. 2) and 0.97 (Fig. 3), the $3 \mathrm{D}$ phase space were found to experience distortion. With a fractional order of 0.97 , the bifurcation diagram of Chen system was found to undergo significant changes (Fig. 4). Without the influence of state-dependent noise, the system is chaotic in the region $36<a<42.5$, however, the introduction of state-dependent noise with intensity $d=1.0$, this regime was found in the range $34<a<44$. The periodic regime $(42.5<a<45)$ and chaotic regime $(45<a<49)$ were not observed when state dependent noise was introduced into the system. Similar distortions were found in the phase space (Fig. 3) and bifurcation (Fig. 5) diagram of the Arneodo-Coullet systems. In the bifurcation diagram of the Arneodo-Coullet system, the period doubling route to chaos was preserved but distorted. The introduction of state-dependent noise was found to suppress chaos in the two systems considered. For increased values of $d$, it is expected that the chaotic state of the system will be destroyed. The inhibition of the period doubling cascade by noise has also been reported in [19]. Intrinsic, although not state-dependent, noise have been found to induce synchronization, coherence and stochastic resonance in the $\mathrm{CO}_{2}$ laser system [36]. The results presented supports several reports in literature which suggests that noise suppress chaos [9-12].

Serletis et al. [37] used extrinsic additive noise to show that stochastic noise changes the dynamics of low dimension chaotic systems. In this work, we have shown that intrinsic additive noise can also shift bifurcation points and produce noise-induced transitions. The impact of external stochastic noise on the phase space of Chen system has been reported [38]. The distortions reported were found to be distinctly different from what was obtained in this report. In this report, the phase space was found to be distorted while Bashkirtseva et al. [38] showed that small extrinsic noise perturbation 
were found to create a stochastic attractor. It was reported in [39] that delayed self-controlling feedback noise caused stabilization of the system, however, this was not obvious in our analysis.

\section{Conclusion}

In this work, we have considered the effect of state dependent noise on the Chen and Arneodo-Coullet chaotic systems. The impact of external noise on the dynamics of chaotic systems have been investigated extensively. The role of internally generated noise fed back into the system has not been given much attention. The noise considered are dependent on feedback from other states of the system as against coloured noise. Hence, they can be regarded as intrinsic noise. The overall effect of state-dependent noise on the system studied is the suppression of chaos. The suppression of chaos by the intrinsic noise considered in this work was done by distortion of the route to chaos and phase space. In the two systems considered, the feedback noise from components of the system was found to break the perioddoubling route to chaos. Thus, this approach will prove useful in the understanding of nonlinearity in systems susceptible to interference from other states of the system. The noise proposed in this study can be used as a convenient way to control chaos in a system using the system variables. Further exploration can be made into the role of intrinsic feedback noise, as considered in this work, on the performance of chaotic systems in secure communication implementation. It will also be interesting to investigate stochastic and vibrational resonance in systems with intrinsic chaotic noise [40].

\section{Compliance with ethical standards}

Conflict of interest The authors declare that they have no competing interests.

\section{Appendix A. Circuit diagrams and simulation}

The circuit representation and resultant phase space are shown here. The analog implementation of the two proposed systems can likewise be generated with possibility of injecting Pseudo-random noise generator. The electronic circuits consists of chain fractance (combined capacitor and resistors), operational amplifiers (TL081CD), multipliers $\left(A_{s}\right)$, resistors and capacitors with power supply unit. Figure 6 display the analog circuit realization of chaotic Chen and Arneodo-Coullet system as well as the phase space representation. 


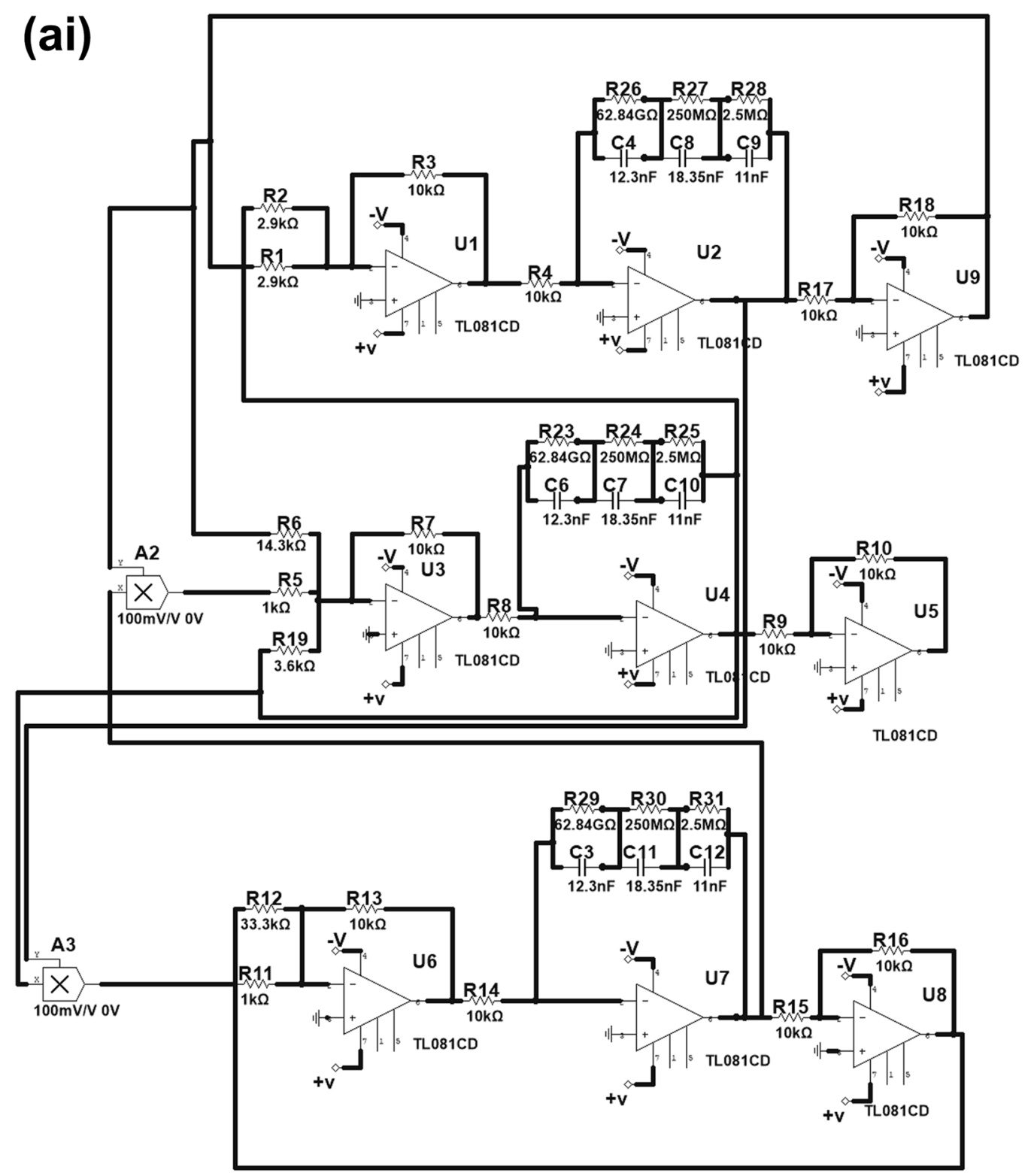

(aii)

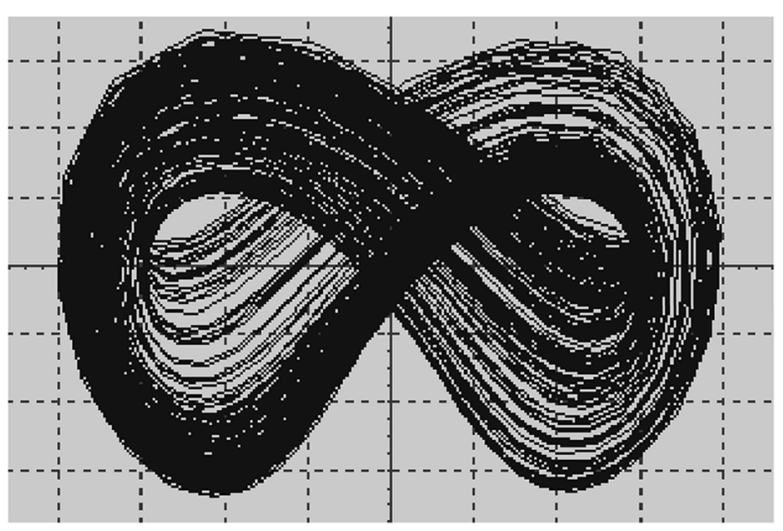

Fig. 6 Circuit diagram and phase space representation for the Chen (Eq. 8) and Arneodo-Coullet (Eq. 9) systems 


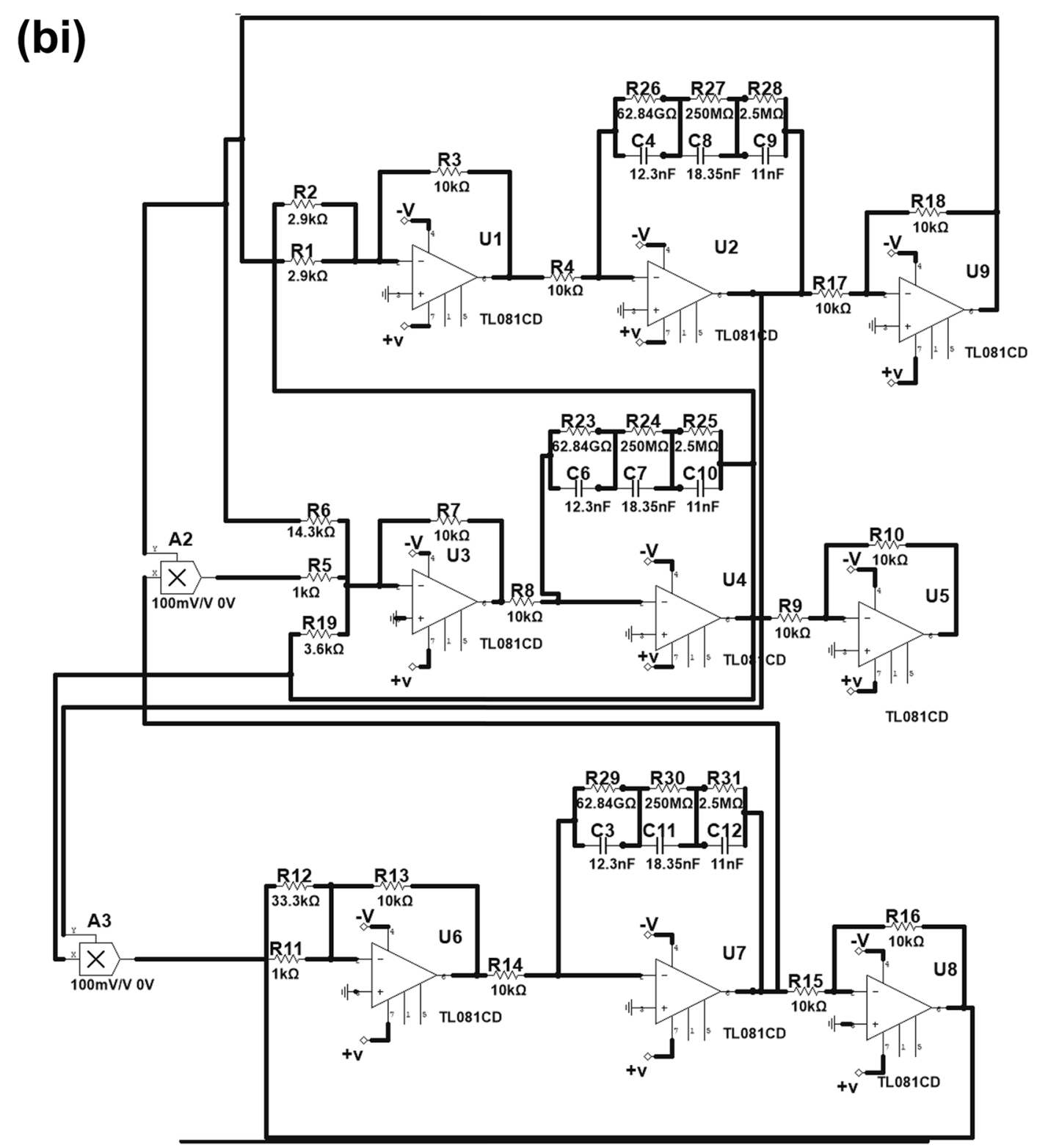

(bii)

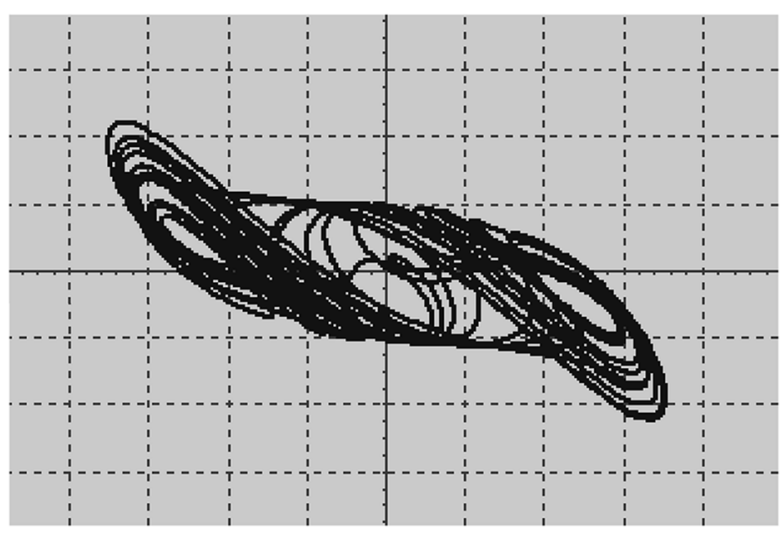

Fig. 6 (continued) 


\section{References}

1. Ogunjo ST, Ojo KS, Fuwape IA (2018) Chapter 15-multiswitching synchronization between chaotic fractional order systems of different dimensions. In: Azar AT, Radwan AG, Vaidyanathan $S$ (eds) Mathematical techniques of fractional order systems. Advances in nonlinear dynamics and chaos (ANDC). Elsevier, Amsterdam

2. Du M, Zaihua $\mathrm{W}, \mathrm{Hu} \mathrm{H}$ (2013) Measuring memory with the order of fractional derivative. Sci Rep 3:3431

3. Ahmed E, El-Sayed AMA, El-Saka HAA (2007) Equilibrium points, stability and numerical solutions of fractionalorder predator-prey and rabies models. J Math Anal Appl 325(1):542-553

4. Chen W-C (2008) Nonlinear dynamics and chaos in a fractionalorder financial system. Chaos Solitons Fractals 36(5):1305-1314

5. Ahmad WM, El-Khazali R (2007) Fractional-order dynamical models of love. Chaos Solitons Fractals 33(4):1367-1375

6. Petras I (2010) Fractional-order memristor-based Chua's circuit. IEEE Trans Circuits Syst II Express Briefs 57(12):975-979

7. Fronzoni L, Giocondo M (1998) Controlling chaos with parametric perturbations. Int J Bifurc Chaos 8(08):1693-1698

8. Astakhov VV, Anishchenko VS, KapitaniakT, Shabunin AV (1997) Synchronization of chaotic oscillators by periodic parametric perturbations. Phys D Nonlinear Phenom 109(1-2):11-16

9. Chacón R (1995) Suppression of chaos by selective resonant parametric perturbations. Phys Rev E 51(1):761

10. Choe C-U, Höhne K, Benner H, Kivshar YS (2005) Chaos suppression in the parametrically driven lorenz system. Phys Rev E 72(3):036206

11. Matsumoto K, Tsuda I (1983) Noise-induced order. J Stat Phys 31(1):87-106

12. Rajasekar S (1995) Controlling of chaotic motion by chaos and noise signals in a logistic map and a Bonhoeffer-van der Pol oscillator. Phys Rev E 51(1):775

13. Dennis B, Desharnais RA, Cushing JM, Henson SM, Constantinno RF (2003) Can noise induce chaos? Oikos 102(2):329-339

14. Lin W, Chen G (2006) Using white noise to enhance synchronization of coupled chaotic systems. Chaos Interdiscip J Nonlinear Sci 16(1):013134

15. Sanchez E, Matias MA, Perez-Munuzuri V (1997) Analysis of synchronization of chaotic systems by noise: an experimental study. Phys Rev E 56(4):4068

16. Wei DQ, Luo XS (2009) Noise-induced chaos in single-machine infinite-bus power systems. EPL (Europhys Lett) 86(5):50008

17. Zhou CS, Kurths J, Allaria E, Boccaletti S, Meucci R, Arecchi FT (2003) Noise-enhanced synchronization of homoclinic chaos in a co 2 laser. Phys Rev E 67(1):015205

18. Qin YH, Li JC (2014) Random parameters induce chaos in power systems. Nonlinear Dyn 77(4):1609-1615

19. Gao JB, Hwang SK, Liu JM (1999) When can noise induce chaos? Phys Rev E 82(6):1132

20. Carrol TL (2005) Chaotic systems that are robust to added noise. Chaos 15:013901

21. Murch AR, Bates RHT (1990) Colored noise generation through deterministic chaos. IEEE Trans Circuits Syst 37(5):608-613

22. Demir K, Ergün S (2018) An analysis of deterministic chaos as an entropy source for random number generators. Entropy 20(12):957
23. Bernstein GM, Lieberman MA (1990) Secure random number generation using chaotic circuits. IEEE Trans Circuits Syst 37(9):1157-1164

24. Liao T-L, Wan P-Y, Yan J-J (2019) Design of synchronized largescale chaos random number generators and its application to secure communication. Appl Sci 9(1):185

25. Hui X, Tong X, Meng X (2016) An efficient chaos pseudo-random number generator applied to video encryption. Optik 127(20):9305-9319

26. Rezk AA, Madian AH, Radwan AG, Soliman AM (2019) Reconfigurable chaotic pseudo random number generator based on fpga. AEU Int J Electron Commun 98:174-180

27. Podlubny I, Petráš I, Vinagre BM, O'leary P, Dorčák L (2002) Analogue realizations of fractional-order controllers. Nonlinear Dyn 29(1-4):281-296

28. Ogunjo ST, Ojo KS, Fuwape IA (2017) Comparison of three different synchronization schemes for fractional chaotic systems. In: Azar AT, Radwan AG, Vaidyanathan S (eds) Fractional order control and synchronization of chaotic systems. Studies in computational intelligence. Springer, Berlin, pp 471-495

29. Dzieliński A, Sierociuk D, Sarwas G (2011) Some applications of fractional order calculus. Bull Pol Acad Sci Tech Sci 58(4):583-592

30. Ahmad WM, Sprott JC (2003) Chaos in fractional-order autonomous nonlinear systems. Chaos Solitons Fractals 16(2):339-351

31. Box GEP, Muller ME (1958) A note on the generation of random normal deviates. Ann Math Stat 29:610

32. Chen G, Ueta T (1999) Yet another chaotic attractor. Int J Bifurc Chaos 09(07):1465-1466

33. Arneodo A, Coullet P, Tresser C (1981) Possible new strange attractors with spiral structure. Commun Math Phys 79(4):573-579

34. Lorenz EN (1963) Deterministic nonperiodic flow. J Atmos Sci 20(2):130-141

35. Rössler OE (1976) An equation for continuous chaos. Phys Lett A 57(5):397-398

36. Zhou CS, Kurths J, Allaria E, Boccaletti S, Meucci R, Arecchi FT (2003) Constructive effects of noise in homoclinic chaotic systems. Phys Rev E 67(6):066220

37. Serletis A, Shahmoradi A, Serletis D (2007) Effect of noise on the bifurcation behavior of nonlinear dynamical systems. Chaos Solitons Fractals 33(3):914-921

38. Bashkirtseva I, Chen G, Ryashko L (2012) Analysis of noiseinduced transitions from regular to chaotic oscillations in the Chen system. Chaos Interdiscip J Nonlinear Sci 22(3):033104

39. Pyragas K (1992) Continuous control of chaos by self-controlling feedback. Phys Lett A 170(6):421-428

40. Adelakun AO, Ogunjo ST, Fuwape IA (2019) Chaos suppression in fractional order systems using state-dependent noise arXiv preprint arXiv, arXiv:1902.05526

Publisher's Note Springer Nature remains neutral with regard to jurisdictional claims in published maps and institutional affiliations. 